\title{
Multiple Invariance ESPRIT
}

\author{
R. Roy, B. Ottersten, L. Swindlehurst, and T. Kailati \\ Information Systems Laboratory \\ Stanford University \\ Stanford, Calif. $9 \$ 305$
}

\begin{abstract}
ESPRIT is a recently developed technique for high-resolution signal parameter estimation. For the specific problem of direction-of-arrival (DOA) estimation, a decrease in computational complexity of orders of magnitude over other high-resolution methods is achieved by exploiting all invariance structure designed into the sensor array. Previously, ESPRIT took advantage of only one such invariance per dimension of the parameter vector. The ubiquitous uniform linear array is an example of a sensor array possessing many such invariances, and the question of which invariance to use naturally arises. In this paper, ESPRIT is extended to address the problem of exploiting all the invariances simultaneously. The nonlinear multiple invariance ESPRIT algorithm is derived and compared via simulation to various standard ESPRIT solutions possible for arrays with multiple invariances.
\end{abstract}

\section{Introduction}

N MANY SIGNAL PROCESSING applications, the objective is to estimate a set of unknown parameters upon which deterministic signals measured by an array of sensors depend. Direction-of-arrival estimation of narrowband sources and detection of exponentials in noise (e.g. linear system identification) are classic examples. These problems naturally possess multi-dimensional geometric characteristics that have only recently been recognized.

In many of the recently developed algorithms (e.g. Schmidt's MUSIC [1], Burg's MEM [2], and Capon's ML [3]) however, the multidimensional nature of the problem is retained only to obtain an estimate of the signal subspace. Once the data have been thus reduced, the multidimensional aspects are eschewed in favor of onedimensional line searches for intersections of estimated signal subspaces and the set of all possible array responses (i.e., the array manifold). Most of the computa-

This work was supported in part by the SDI/IST Program managed by the Office of Naval Research under Contract No001485-K-0550, and by the Joint Services Program at Stanford University (US Army, US Navy, US Air Force) under Contract DAALO388-C-0011. tional effort involved in these algorithms is expended in performing these one-dimensional searches.

A robust, computationally efficient technique known as ESPRIT [4] has recently been developed. It mitigates most of the computational complexity of previous high resolution techniques by exploiting a displacement invariance designed into the sensor array. ESPRIT also manifests lower sensitivity to array errors than previous high resolution methods, and, through use of a total leastsquares (TLS) minimization criterion, yields apparently unbiased parameter estimates even at low SNRs.

In many situations, however, the sensor array possesses more than one displacement invariance. The ubiquitous uniform linear array is a prime example. For such arrays, the standard ESPRIT algorithm is applied by first selecting a pair of subarrays that satisfy one of the many inherent invariances. The question naturally arises as to whether or not there is an optimal choice of subarrays. More fundamentally, the issue of the proximity of any standard ESPRIT solution to the optimal solution that exploits all the invariances simultaneously is most certainly relevant. These are the questions to which this paper is addressed. In the following section, three equivalent formulations of the standard TLS ESPRIT solution are presented. Though certainly of pedagogical interest, the equivalence also demonstrates how 'TLS estimates of the array manifold vectors may be obtained, and provides a framework for extending ESPRIT to arrays with multiple invariances. The extension of the algorithm to such arrays is elucidated in Section 3, and some simulation results are given in Section 4 .

\section{TLS ESPRIT}

In the standard TLS ESPRIT problem [4], the sensor array is assumed to be composed of two identical subarrays separated by a fixed displacement vector $\Delta$. For such arrays, the manifold of steering vectors has the form $\mathbf{A}_{e}=\left[\mathbf{A}^{\boldsymbol{T}} \boldsymbol{\Phi}^{T_{A}} \mathbf{A}^{T}\right.$, where $\mathbf{A}$ is $m \times d$ for $m$ sensors (per subarray) and $d$ sources, and where $\Phi$ is a diagonal matrix. For the DOA estimation problem, the diagonal elements of $\Phi$ are given by $\exp \left\{-j 2 \pi \Delta \sin \theta_{i} / c\right\}, i=$ 
$1, \cdots, d$, where $c$ is the wave propagation speed and $\theta_{i}$ is the direction-of-arrival (DOA) of the $i^{\text {th }}$ source. For notational purposes, we partition the $d$ "signal" eigenvectors $\mathbf{E}$ of the array covariance matrix in the same fashion as $\mathbf{A}_{e}$, and note that, in the absence of noise, a full rank $d \times d$ matrix $T$ would exist such that

$$
\mathbf{E}=\left[\begin{array}{l}
\mathbf{E}_{\boldsymbol{X}} \\
\mathbf{E}_{\mathbf{Y}}
\end{array}\right]=\left[\begin{array}{c}
\mathbf{A} \\
\mathbf{A} \mathbf{\Phi}
\end{array}\right] \mathbf{T} .
$$

The fundamental result of the TLS ESPRIT algorithm is that $\Phi$ can be estimated as the eigenvalues of a matrix $\boldsymbol{\Psi}$ obtained from the following minimization problem:

Given subspace estimates $\mathbf{E}_{x}$ and $\mathbf{E}_{Y}$, find a matrix $\mathbf{F} \in \mathbb{C}^{2 d \times d}$ to minimize

$$
J=\|\left[\mathbf{E}_{x}\left\lfloor\mathbf{E}_{r}\right] \mathbf{F} \|_{F}^{2}\right.
$$

subject to

$$
\mathbf{F}^{*} \mathbf{F}=\mathbf{I}
$$

where

$$
\begin{aligned}
& \mathbf{F} \stackrel{\text { def }}{=}\left[\begin{array}{l}
\mathbf{F}_{\mathbf{x}} \\
\mathbf{F}_{\boldsymbol{Y}}
\end{array}\right], \\
& \boldsymbol{\Psi}=-\mathbf{F}_{\boldsymbol{X}}\left[\mathbf{F}_{Y}\right]^{-1} .
\end{aligned}
$$

The above minimization problem is not, however, the most common statement of the TLS linear parameter estimation problem. In [5], for example, the problem is posed in the following manner:

Given $\mathbf{C}, \mathbf{D} \in \mathbb{C}^{m \times d}, m>d$, find $\mathbf{R}_{\mathbf{C}}$ and $\mathbf{R}_{\mathbf{D}}$ of minimum Frobenius ${ }^{2}$ norm, and $\mathrm{X}$ such that

$$
\left[\mathbf{C}+\mathbf{R}_{C}\right] \mathbf{X}=\mathbf{D}+\mathbf{R}_{\boldsymbol{D}} .
$$

Note that in general $D$ need not have the same number of columns as $\mathbf{C}$ though for the applications described herein, $\mathbf{C}$ and $\mathbf{D}$ have the same dimensions and $\mathbf{X}$ is square. The connection with the original formulation of TLS ESPRIT given above can be obtained by noting that $\mathbf{R}_{C}$ and $\mathbf{R}_{D}$ represent errors in the subspace estimates $\mathbf{E}_{\boldsymbol{x}}=\mathbf{C}$ and $\mathbf{E}_{\boldsymbol{Y}}=\mathbf{D}$ respectively, and that $\mathbf{X}$ is the operator $\boldsymbol{\Psi}$ whose eigenvalues are the parameters of interest. Thus, the TLS ESPRIT estimation problem can also be stated as follows:

Given subspace estimates $\mathbf{E}_{x}$ and $\mathbf{E}_{Y}$, find an operator $\mathbf{\Psi}$ and residual matrices $\mathbf{R}_{E_{X}}, \mathbf{R}_{E_{Y}}$ to minimize

$$
J=\left\|\left[\mathbf{R}_{E_{X}} \mid \mathbf{R}_{E_{Y}}\right]\right\|_{F}^{2}
$$

2If knowledge of the covariance of the errors is available (in general in the form of a 4-tensor), a weighted Frobenius norm can be employed. Since for the problems considered herein it can be argued that the errors are independent and identically distributed (at least asymptotically), the weighting matrix or metric is proportional to the identity. subject to

$$
\left[\mathbf{E}_{\boldsymbol{X}}+\mathbf{R}_{E_{X}}\right] \mathbf{\Psi}=\mathbf{E}_{Y}+\mathbf{R}_{E_{Y}} .
$$

A further reformulation of this constrained minimization problem provides the framework for generalizing TLS ESPRIT to arrays with multiple invariances. Starting with the TLS formulation in equations (7) and (8), define $\mathbf{B} \stackrel{\text { def }}{=} \mathbf{E}_{\boldsymbol{x}}+\mathbf{R}_{\boldsymbol{E}_{\boldsymbol{X}}}$. Substituting into equation (8) gives $\mathbf{E}_{Y}+\mathbf{R}_{E_{Y}}=\mathbf{B} \boldsymbol{\Psi}$. Rearranging these equations gives

$$
\begin{aligned}
& \mathbf{R}_{E_{X}}=\mathbf{E}_{\boldsymbol{x}}-\mathbf{B}, \\
& \mathbf{R}_{E_{Y}}=\mathbf{E}_{\boldsymbol{Y}}-\mathbf{B} \boldsymbol{\Psi},
\end{aligned}
$$

so the minimization becomes

$$
\min _{\{\mathbf{B}, \mathbf{\Psi}\}} J=\min _{\{\mathbf{B}, \mathbf{\Psi}\}}\left\|\left[\begin{array}{l}
\mathbf{E}_{\boldsymbol{X}} \\
\mathbf{E}_{Y}
\end{array}\right]-\left[\begin{array}{c}
\mathbf{B} \\
\mathbf{B} \mathbf{\Psi}
\end{array}\right]\right\|_{F}^{2} .
$$

An important point to be made here is that the minimization of equation (11) is nothing more than a leastsquares fit to the model of equation (1), with $\mathbf{B}=\mathbf{A T}$ and $\boldsymbol{\Psi}=\mathbf{T}^{-1} \mathbf{\Phi} \mathbf{T}$. This formulation of the problem is much clearer than that given in equations (2) through (5) for at least two reasons. First, it is obvious from (11) why $\Phi$ is given as the eigenvalues of the estimated operator $\mathbf{\Psi}$. Secondly, the question of how to naturally incorporate multiple displaced subarrays into the problem is easily answered, as will be explained in the following section. A proof formally establishing the equivalence of the minimization problems posed in equation (11) and in equations (2) through (5) can be found in the appendix.

\section{Multiple Invariance ESPRIT}

Using the alternative formulation of the minimization as given in equation (11), the standard ESPRIT solution can be easily extended to arrays with multiple invariances. Assuming the presence of $p$ subarrays, each with its own displacement vector $\Delta_{i}$, the multiple invariance ESPRIT problem can be stated as follows:

Given $p$ subspace estimates $\mathbf{E}_{0}, \mathbf{E}_{1}, \ldots, \mathbf{E}_{p-1} \in$ $\mathbb{C}^{m \times d}$, find an operator $\Psi \in \mathbb{C}^{d \times d}$ and a sub. space estimate $\mathrm{B} \in \mathbb{C}^{m \times d}$ to minimize

$$
J=\left\|\left[\begin{array}{l}
\mathbf{E}_{0}-\mathbf{B} \\
\mathbf{E}_{1}-\mathbf{B} \mathbf{\Psi} \\
\mathbf{E}_{2}-\mathbf{B} \boldsymbol{\Psi} \frac{\Delta_{1}}{\partial_{1}} \\
\vdots \\
\mathbf{E}_{p-1}-\mathbf{B} \Psi^{\frac{\Delta_{p-1}}{\Delta_{1}}}
\end{array}\right]\right\|_{F}^{2} .
$$

Note that the $\Delta_{i}$ 's are collinear, though not necessarily of equal magnitude. 
The estimates of the parameters of interest are again given as the eigenvalues of the operator $\mathbf{\Psi}$. For the special case of a uniform linear array, $\Delta_{i}=i \Delta_{1} \quad \forall i=$ $1, \ldots, p-1$, and the cost function becomes

$$
J=\left\|\left[\begin{array}{l}
\mathbf{E}_{0}-\mathbf{B} \\
\mathbf{E}_{1}-\mathbf{B} \mathbf{\Psi} \\
\mathbf{E}_{2}-\mathbf{B} \boldsymbol{\Psi}^{2} \\
\vdots \\
\mathbf{E}_{p-1}-\mathbf{B} \boldsymbol{\Psi}^{p-1}
\end{array}\right]\right\|_{F}^{2}
$$

Note that in this case $p$ is the number of sensors and $m=1$.

The minimization of $J$ over $\left\{\Psi \in \mathbb{C}^{d \times d}, \mathbf{B} \in \mathbb{C}^{m \times d}\right\}$ is a nonlinear, computationally complex problem. A somewhat simpler expression for $J$ can be obtained by solving for $\mathbf{B}$ explicitly in terms of $\boldsymbol{\Psi}$. Defining

$$
\begin{aligned}
& \mathbf{E} \stackrel{\text { def }}{=}\left[\mathbf{E}_{0}\left|\mathbf{E}_{1}\right| \cdots: \mathbf{E}_{p-1}\right], \\
& \overline{\boldsymbol{\Psi}} \stackrel{\text { def }}{=}\left[\mathbf{I} \mid \Psi: \Psi^{\frac{\Delta_{2}}{\Delta_{1}}}: \cdots: \Psi^{\frac{\Delta_{k-1}}{\Delta_{1}}}\right]
\end{aligned}
$$

and using standard properties of the trace operator, the cost function becomes

$$
J=\operatorname{Tr}\left\{[\mathbf{E}-\mathbf{B} \overline{\mathbf{\Psi}}]^{*}[\mathbf{E}-\mathbf{B} \overline{\mathbf{\Psi}}]\right\} .
$$

Solving $\partial_{\mathbf{B}} J=0$ yields

$$
\mathbf{B}=\mathbf{E} \overline{\mathbf{\Psi}}^{*}\left[\overline{\mathbf{\Psi}} \overline{\mathbf{\Psi}}^{*}\right]^{-1}
$$

which, when substituted for $\mathbf{B}$ in the expression of (3) for the cost function $J$, gives

$$
J=\operatorname{Tr}\left\{\mathbf{E}^{*} \mathbf{E}\left(\mathbf{I}-\overline{\mathbf{\Psi}}^{*}\left[\overline{\mathbf{\Psi}} \overline{\mathbf{\Psi}}^{*}\right]^{-1} \overline{\mathbf{\Psi}}\right)\right\},
$$

which must now be minimized over $\boldsymbol{\Psi}$. For the specific case of a uniform linear array, the partial derivative of $J$ with respect to $\Phi$ can be succinctly written as

$$
\partial_{\mathbf{\Psi}} J=\tilde{\mathbf{\Psi}} \mathbf{C} \overline{\mathbf{\Psi}}^{*},
$$

where

$$
\tilde{\mathbf{\Psi}} \stackrel{\text { def }}{=}\left[\mathbf{I}\left|\mathbf{\Psi}^{*}\right| \mathbf{\Psi}^{* 2}|\cdots| \mathbf{\Psi}^{*(p-1)}\right],
$$

and where $\mathbf{C}$ is an upper-left triangular block Hankel matrix whose first block row is given by $\left[\mathbf{C}_{1}\left|\mathbf{C}_{2}\right| \ldots \mid \mathbf{C}_{p}\right]$, with $\mathbf{C}_{i} \stackrel{\text { def }}{=} \mathbf{B}^{*} \mathbf{B} \Psi^{i}-\mathbf{E}_{i}^{*} \mathbf{B}$. Provided that $\Delta_{i} / \Delta_{1}$ is rational, the partial derivative $\partial_{\Psi} J$ can also be obtained for the more general multiple invariance case, though the resulting expressions are quite cumbersome. .

Since a closed form expression for $\Psi$ is not possible, some type of search technique is required to achieve $\partial_{\Psi} J=0$. Fortunately, the original single invariance TLS ESPRIT algorithm can be applied to produce an excellent initial estimate as a starting point for a simple gradient search. The multiple invariance ESPRIT algorithm is thus implemented as follows:
1. Using the TLS ESPRIT algorithm, obtain an initial estimate of the operator $\hat{\mathbf{\Psi}}(0)$. Obtain $\hat{\mathbf{B}}(0)$ using (12). Repeat steps 2 and 3 until convergence $\left(\left\|\partial_{\mathbf{\Psi}} J\right\| \approx 0\right)$.

2. Update $\hat{\boldsymbol{\Psi}}(k+1)=\hat{\boldsymbol{\Psi}}(k)+\alpha \partial_{\mathbf{\Psi}} J(k)$

3. Set $\hat{\mathbf{B}}(k+1)=\mathbf{E} \overline{\boldsymbol{\Psi}}(k)^{*}\left[\overline{\mathbf{\Psi}}(k) \overline{\mathbf{\Psi}}^{*}(k)\right]^{-1}$, and evaluate $\left\|\partial_{\mathbf{\Psi}} J(k+1)\right\|$.

\section{Simulation Results}

To investigate the performance improvement of the iterated ESPRIT algorithm over the single invariance $\boldsymbol{E S}$ PRIT algorithm, simulations were conducted using a 12 element uniform linear array (ULA). The array was split into varying numbers of subarrays, in each case using every element once and only once. Though there are a wide variety of such arrays, they can be grouped into sets of arrays that have the same $\Delta$ for rational comparison of the results between estimates from different subarray structures.

Maintaining $\Delta=1$ (in normalized units of the ULA element separation), there are 6 ways of decomposing the 12 element ULA into subarrays. If elements in a subarray are indicated by \{\} , corresponding elements in the various subarray sets are assumed to be identical and separated by $\Delta$. Thus, the decomposition into 2 subarrays is indicated by $\{1,3,5,7,9,11\},\{2,4,6,8,10,12\}$ where the elements of the ULA have been labeled from 1 to 12 . Elements 1 and 2 are therefore identical and separated by $\Delta$ as are 3 and 4,5 and 6 , etc.. For this decomposition there is only one invariance. The decomposition into three subarrays, with two invariances, is given by $\{1,4,7,10\},\{2,5,8,11\},\{3,6,9,12\}$, where now elements 1,2 , and 3 are assumed to he identical and form a threr element uniform linear subarray with element separation $\Delta$. The decomposition into 4,6 and finally 12 subarrays should now be easily seen. For the latter case, each subarray is composed of a single element of the underlying ULA, and for this case iterated ESPRIT is exploiting the entire invariance structure of the array.

For each of the decompositions, the single invariance ESPRIT results were generated using maximally overlapping subarrays with $\Delta=1$. For the 3 subarray decomposition, this corresponds to combining subarrays 1 and 2 into a single subarray and subarrays 2 and 3 into the second subarray. Four elements are shared in common. The ESPRIT estimates were also used as initial conditions for the iterations. Due to the manifestly unbiased nature of the ESPRIT estimates, the iteration converged quite rapidly. With convergence criterion $\left\|\partial_{\Psi} J(k+1)\right\|_{F}<10^{-4}$, the average number of iterations for 2,3 , and 5 invariances was 7 , and for 11 invarances, 21 iterations were required on average. 
The results shown in Figures 1 through 5 are for a scenario in which two sources are present at electrical angles $(\phi=2 \pi \Delta \sin \theta / \lambda)$ of $0.17 \pi$ and $0.22 \pi$ radians. The SNR per element was assumed to be $0 \mathrm{~dB}$ for both sources and the sources were uncorrelated. The number of snapshots in each trial was 100 , and 1000 Monte Carlo trials were run. The quantities shown in Figures 1 through 2 are the magnitude of the mean of the error in the complex pole location over the 1000 trials as a function of the number of invariances and its standard deviation. From Figure 3 it can be seen that the bias in the mean is negligible in both ESPRIT and iterated ESPRIT. Since iterated ESPRIT reduces to ESPRIT when there is only one invariance to exploit, the results are identical. As the number of invariances increases to the maximum of 11 , a gradual improvement of up to $30 \%$ is achieved. It should be noted however, that for the 12 element subarray, there is a significant latitude in the choice of $\Delta$ for ESPRIT and simulations indicate that minimum variance estimates are obtained for $\Delta$ 's near half the maximum possible (again using all the elements once and only once), in this case $\Delta=3$.

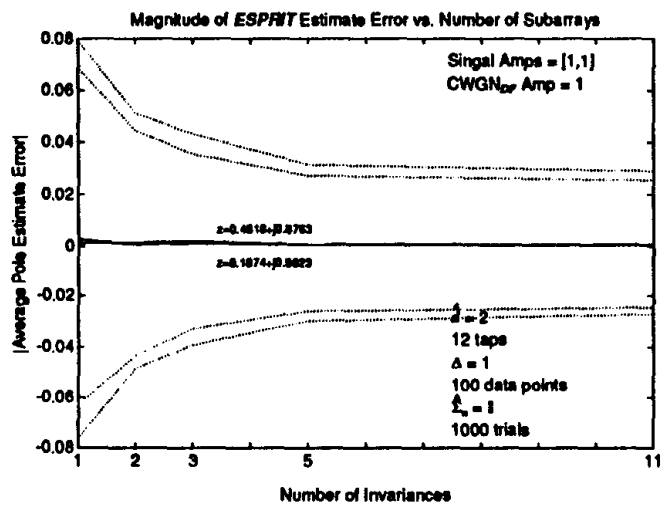

Figure 1: Bias and Standard Deviation of ESPRIT Estimates versus Number of Invariances

In Figures 4 and 5, standard and iterated ESPRIT are compared with root-MUSIC, an analytic version of the well-known MUSIC algorithm applicable to uniform linear arrays and constant amplitude plane waves. The standard ESPRIT results are obtained from maximally overlapping 11-element subarrays, while iterated ESPRIT is exploiting all 11 invariances present. The bias in the root-MUSIC estimates is easily seen. As discussion of root-MUSIC and the inherent biases in the algorithm can be found in [4].

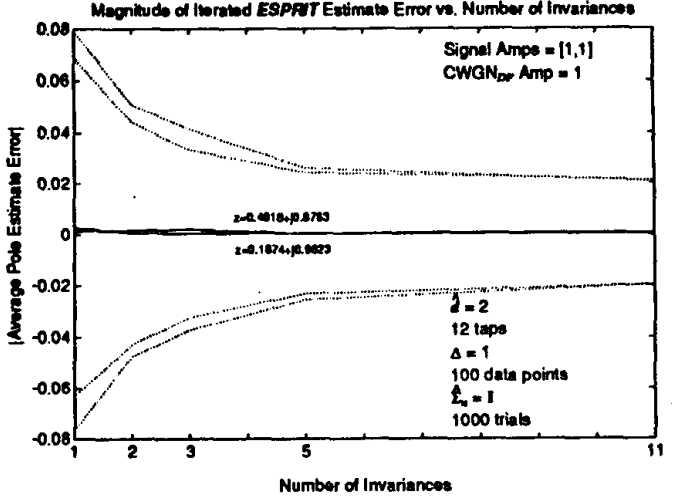

Figure 2: Bias and Standard Deviation of Iterated ESPRIT Estimates versus Number of Invariances

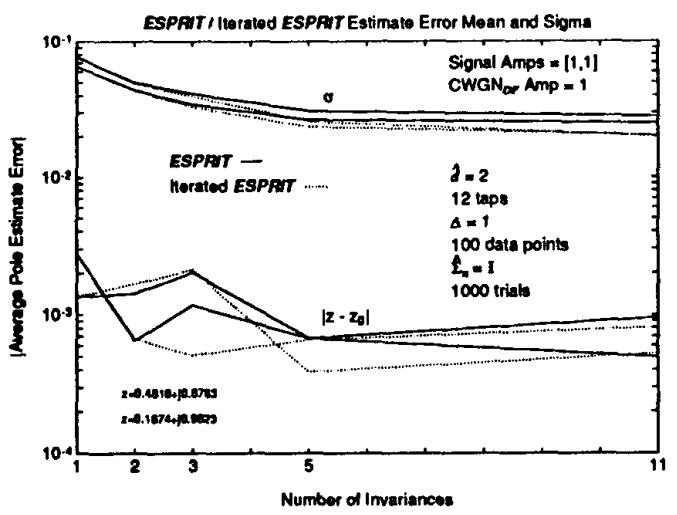

Figure 9: Comparison of ESPRIT and Iterated ESPRIT Estimate Bias and Standard Deviation

\section{Concluding Remarks}

In this paper, an extension of the TLS ESPRIT algorithm to arrays with multiple one-dimensional invariances has been presented. It was shown how a simple reformulation of the TLS minimization problem leads to a framework into which a given multiple invariance structure can be easily incorporated. One drawback of using the algorithm to exploit such invariances is that a closed-form solution to the problem can not be obtained. However, using the standard TLS ESPRIT estimate as a starting value, a simple gradient search technique converges rapidly to the desired solution. To date, simulations performed for a variety of scenarios indicate that exploiting the full invariance structure of an array offers some improvement in performance (reduction in estimate error variance) over the standard single invariance $E S$ PRIT solution. The performance of the standard ES- 


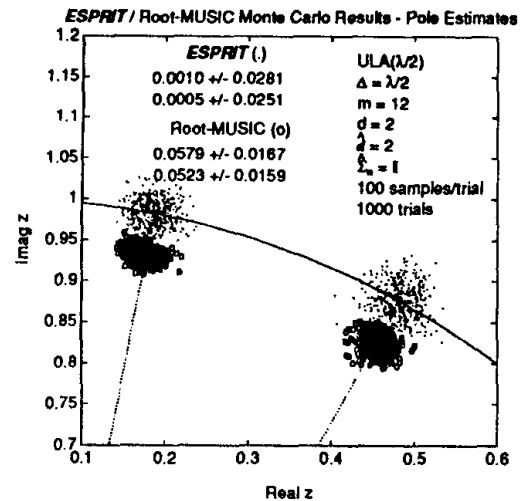

Figure 4: ESPRIT and Root-MUSIC Estimated Pole Locations

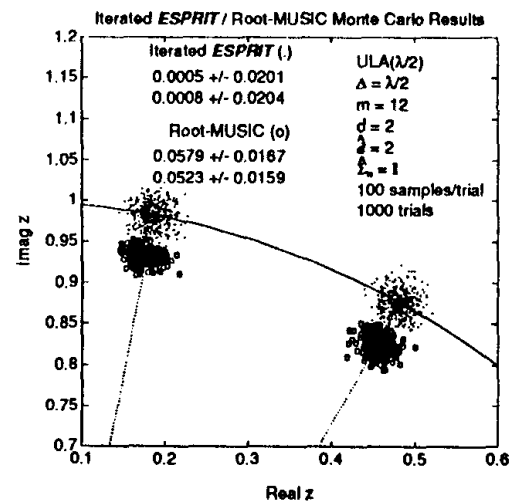

Figure 5: Iterated ESPRIT and Root-MUSIC Estimated Pole Locations

PRIT algorithm has proved to be remarkably similar to that of the multiple invariance version however.

\section{Appendix}

\section{Equivalence of Two Forms of the TLS ESPRIT Problem}

Here the equivalence between the operator $\Psi$ obtained from equations (2) through (5) and that obtained from equation (11) is established. Using standard properties of the trace operator, the minimization of equation (2) can be rewritten as

$$
\min _{\mathbf{F}} J=\min _{\mathbf{F}} \operatorname{Tr}\left\{\mathbf{E}_{x Y}^{*} \mathbf{E}_{x Y} P_{F}\right\}
$$

where

$$
\mathbf{E}_{\boldsymbol{X} Y} \stackrel{\text { def }}{=}\left[\mathbf{E}_{\boldsymbol{x}}\left[\mathbf{E}_{\boldsymbol{Y}}\right]\right. \text {, }
$$

and where $P_{F}=\mathbf{F}\left[F^{*} \mathbf{F}\right]^{-1} \mathbf{F}^{*}=\mathbf{F F}^{*}$ is the projection onto the (full rank) columns of F. It is shown in [4] that the TLS ESPRIT estimate of $\boldsymbol{\Psi}$ for this formulation of the problem is given by $\Psi=-\mathbf{E}_{12} \mathbf{E}_{22}^{-1}$, where

$$
\mathbf{E}_{X Y}^{*} \mathbf{E}_{x Y}=\left[\begin{array}{ll}
\mathbf{E}_{11} & \mathbf{E}_{12} \\
\mathbf{E}_{21} & \mathbf{E}_{22}
\end{array}\right] \Lambda\left[\begin{array}{ll}
\mathbf{E}_{11} & \mathbf{E}_{12} \\
\mathbf{E}_{21} & \mathbf{E}_{22}
\end{array}\right]^{*}
$$

is the eigendecomposition of $\mathbf{E}_{\boldsymbol{X} Y}^{*} \mathbf{E}_{X Y}$.

Returning to the expression of equation (13), we see that the minimization problem of (11) may be rewritten as

$$
\min _{\mathbf{\Psi}} J=\min _{\mathbf{\Psi}} \operatorname{Tr}\left\{\mathbf{E}_{x \boldsymbol{Y}}^{*} \mathbf{E}_{\boldsymbol{x} Y} P_{\underline{\mathbf{\Psi}} \cdot}\right\}
$$

The two minimization problems are thus manifestly equivalent ${ }^{3}$ since both 1 are minimizations of the same functional form over rank $d$ projection matrices in $\mathbb{C}^{2 d \times 2 d}$. To see that the operator $\Psi$ obtained from (13) is identical to that obtained from the original formulation, note that by the orthogonality of the eigenvectors of $\mathbf{E}_{X Y}^{*} \mathbf{E}_{X Y}, P_{\tilde{\mathbf{\Psi}} \cdot}^{\perp}=P_{\mathbf{E}_{2}}$ implies $P_{\overline{\mathbf{\Psi}} \cdot}=P_{\mathbf{E}_{1}}$. Therefore there exists a non-singular matrix $\mathbf{T}$ such that

$$
\left[\begin{array}{l}
\mathbf{I} \\
\mathbf{\Psi}^{*}
\end{array}\right]=\left[\begin{array}{l}
\mathbf{E}_{11} \\
\mathbf{E}_{21}
\end{array}\right] \mathbf{T} \text {. }
$$

The estimate of $\boldsymbol{\Psi}$ is thus given by $\mathbf{E}_{11}^{-*} \mathbf{E}_{21}^{*}$, and the orthogonality relation

$$
\mathbf{E}_{11}^{*} \mathbf{E}_{12}+\mathbf{E}_{21}^{*} \mathbf{E}_{22}=0
$$

now easily establishes the equivalence of the two estimates of $\mathbf{\Psi}$ as was to be shown.

\section{References}

[1] R. O. Schmidt, A Signal Subspace Approach to Multiple Emitter Location and Spectral Estimation, PhD thesis, Stanford University, Stanford, CA., 1981.

[2] J. P. Burg, Maximum Entropy Spectral Analysis, $\mathrm{PhD}$ thesis, Stanford University, Stanford, CA., 1975.

[3] J. Capon, High resolution frequency wave number spectrum analysis, Proc. IEEE, 57:1408-1418, 1969.

[4] R. H. Roy, ESPRIT - Estimation of Signal Parameters via Rotational Invariance Techniques, PhD thesis, Stanford University, Stanford, CA., 1987.

[5] G. H. Golub and C. F. Van Loan, Matrix Comptutations, Johns Hopkins University Press, Baltimore, MD., 1984.

\footnotetext{
${ }^{3}$ There is a subtle difference in the constraints however. Math ematically speaking, the set of matrices of the form $\Psi^{*}$ is a subset of all full-rank matrices in $\mathbb{E}^{2 d x d}$ since full-rank matrices whose upper $d \times d$ block is not invertible can not be converted into the form of $\mathbf{\psi}^{*}$. For the problems considered herein, this set has zero probability of occurring and need not be considered further.
} 